\title{
Genetic diversity and structure of the threatened species Sinopodophyllum hexandrum (Royle) Ying
}

\author{
W. Liu ${ }^{1,2 *}$, J. Wang ${ }^{1 *}$, D.X. Yin ${ }^{3}$, M. Yang ${ }^{4}$, P. Wang ${ }^{1}$, Q.S. Han ${ }^{1}$, Q.Q. Ma ${ }^{1}$, \\ J.J. Liu ${ }^{5}$ and J.X. Wang ${ }^{6}$ \\ ${ }^{1}$ College of Forestry, Northwest A\&F University, Yangling, China \\ ${ }^{2}$ Agricultural College, Henan University of Science and Technology, \\ Luoyang, China \\ ${ }^{3}$ College of Agricultural Engineering, \\ Henan University of Science and Technology, Luoyang, China \\ ${ }^{4}$ College of Arts, Changzhou University, Changzhou, China \\ ${ }^{5}$ College of Landscape Architecture and Arts, Northwest A\&F University, \\ Yangling, China \\ ${ }^{6}$ College of Natural Resources and Environment, Northwest A\&F University, \\ Yangling, China \\ *These authors contributed equally to this study. \\ Corresponding authors: J.J. Liu / J.X. Wang \\ E-mail: 1jj@nwsuaf.edu.cn / jwang118@126.com \\ Genet. Mol. Res. 15 (2): gmr.15028130 \\ Received December 17, 2015 \\ Accepted January 15, 2016 \\ Published June 10, 2016 \\ DOI http://dx.doi.org/10.4238/gmr.15028130
}

\begin{abstract}
Sinopodophyllum hexandrum is an important medicinal plant that has been listed as an endangered species, making the conservation of its genetic diversity a priority. Therefore, the genetic diversity and population structure of $S$. hexandrum was investigated through inter-simple sequence repeat analysis of eight natural populations. Eleven selected primers generated 141 discernible fragments. The percentage of polymorphic bands was $37.59 \%$ at the species level, and $7.66-24.32 \%$ at the population level. Genetic
\end{abstract}


diversity of $S$. hexandrum was low within populations (average $H_{\mathrm{E}}=$ $0.0366)$, but higher at the species level $\left(H_{\mathrm{E}}=0.0963\right)$. Clear structure and high genetic differentiation were detected between populations using unweighted pair groups mean arithmetic and principle coordinate analysis. Clustering approaches clustered the eight sampled populations into three major groups, and AMOVA confirmed there to be significant variation between populations $(63.27 \%)$. Genetic differentiation may have arisen through limited gene flow $\left(\mathrm{N}_{\mathrm{m}}=0.3317\right)$ in this species. Isolation by distance among populations was determined by comparing genetic distance versus geographical distance using the Mantel test. The results revealed no correlation between spatial pattern and geographic location. Given the low within-population genetic diversity, high differentiation among populations, and the increasing anthropogenic pressure on this species, in situ conservation measures, in addition to sampling and ex situ preservation, are recommended to preserve $S$. hexandrum populations and to retain their genetic diversity.

Key words: Sinopodophyllum hexandrum; Genetic diversity; Population structure; Conservation

\section{INTRODUCTION}

Sinopodophyllum hexandrum (Royle) Ying, or Himalayan may apple, is an endangered perennial herb belonging to the family Berberidaceae (Editorial Committee of Flora of China, 2011). It is distributed in the alpine Himalayan regions at elevations ranging from 2700 to 4500 $\mathrm{m}$ (Ma and Hu, 1996). In China, S. hexandrum, the only species of this genus, is mainly found growing wild in Sichuan Province, Yunnan Province, Tibet Autonomous Region, Shaanxi Province, Gansu Province, Qinghai Province, and Ningxia Province (Ma and Hu, 1996).

The plant kingdom has provided us with many important medicaments, including anticancer agents (Cragg et al., 1997), and traditional Chinese medicine has contributed to the identification of these substances. As a component of traditional Chinese medicine, $S$. hexandrum has been used in folk medicine by local populations. The roots of $S$. hexandrum contain high levels of lignans, including podophyllotoxin, 4'-demethylpodophyllotoxin, and dehydropodophyllotoxin, at levels up to 3-fold higher than are found in the American species Podophyllum peltatum (Giri and Lakshmi, 2000). Although the aryltetralin lignan, podophyllotoxin, is the most important lignan for human health, it is also the most cytotoxic, because it is a precursor for the semi-synthesis of the anticancer pharmaceuticals etoposide (VP-16), teniposide (VM-26), GP-7, NK-611, etopophos, GL-331, and TOP-53 (Giri and Lakshmi, 2000). These chemotherapeutic drugs have been used in the treatment of cancers, including lung, cervical, and testicular cancers, and neuroblastoma, hepatoma, and certain types of leukemia (Giri and Lakshmi, 2000). In addition to its role in anti-cancer drugs, podophyllotoxin is the precursor of $\mathrm{CPH}-82$, which is currently being tested in clinical trials in Europe for the treatment of rheumatoid arthritis (Svensson and Pettersson, 2003). However, due to its medicinal importance, wild $S$. hexandrum has been extensively harvested as it represents the commercial source of podophyllotoxin. The destructive harvest of these plants has resulted in $S$. hexandrum being added to the endangered species list of the Convention 
on International Trade in Endangered Species of Wild Fauna and Flora (Lata et al., 2010). S. hexandrum was classified as an endangered species (grade 3) in 1987 by the Chinese Plant Red Book (Fu, 1991).

Currently, with enhanced awareness of its medicinal value and clinical efficacy, the wild population of $S$. hexandrum in China has been noted to be very small and declining rapidly. As a result, the availability of podophyllotoxin from plants is limited due to intense harvesting, habitat fragmentation, low rate of natural regeneration, and a lack of organized cultivation. Without timely and effective measures to protect wild populations of $S$. hexandrum, it could become extinct, subsequently depriving humans a drug that may be used to treat cancer.

Knowledge on genetic diversity at intraspecific levels is an important prerequisite for species conservation and rational exploitation programs. However, previous studies have focused on determining the chemical components (Giri and Lakshmi, 2000; Zhao et al., 2011), biological properties (Chattopadhyay et al., 2004), micropropagation (Nadeem et al., 2000), and phylogenetic evolution (Li et al., 2011) of this species. To date, limited publications have characterized the genetic diversity of $S$. hexandrum, and only studies in populations from the Northwestern Himalayan region are available (Alam et al., 2008; Alam et al., 2009; Naik et al., 2010). Of note, the genetic diversity of this species was investigated in populations from western Sichuan Province and Qinling Mountains in China, using molecular marker techniques (Xiao et al., 2006a, 2006b; Liu et al., 2014).

Molecular markers are very useful tools in the study of genetic diversity. The ISSR marker technique is particularly suited to the analysis of genetic polymorphisms in species without available sequence information (Bornet and Branchard, 2001). Studies on the genetic diversity and conservation of $S$. hexandrum populations in China are needed, because climate change and local overexploitation may further endanger this species. We used ISSR markers to investigate the genetic diversity and structure of $S$. hexandrum from representative production regions throughout China, including Ningxia, Shaanxi, Qinghai, Gansu, Sichuan, Yunnan, and Tibet, where historical records have confirmed it has grown naturally. The present study also aimed to establish management strategies for the conservation genetics of $S$. hexandrum.

\section{MATERIAL AND METHODS}

\section{Plant materials}

Twenty plants from each of eight wild $S$. hexandrum populations were collected for subsequent DNA analysis between July 19, 2014 and September 17, 2014 (Figure 1). The distance between adjacent samples was at least $5 \mathrm{~m}$ to increase the likelihood of sampling inter-individual variation within each population (Xiao et al., 2006a,b). In total, 160 individual samples were collected. About 2-10 g of fresh young leaves per plant was immediately frozen in liquid nitrogen and then stored at $-80^{\circ} \mathrm{C}$. Key information about the $S$. hexandrum populations studied is summarized in Table 1. Voucher specimens from all populations were identified by Professor Jianjun Liu of Northwest A\&F University, and were deposited at the Herbarium of Northwest A\&F University (WUK0780791-0780798).

\section{DNA extraction}

Total genomic DNA was extracted from frozen leaves using the Plant Genomic DNA 
Rapid Extraction kit (Spin-column), which is designed to isolate genomic DNA from a variety of limited plant samples (BioTek Corporation, Beijing, China). The DNA was quantified by comparing with diluted Lambda DNA in 1\% agarose gel (Gene Genius Bio Imaging System, Syngene); and the extracted DNA was diluted in TE buffer to a final concentration of $50 \mathrm{ng} /$ $\mathrm{mL}$ and stored at $-20^{\circ} \mathrm{C}$ until further use.

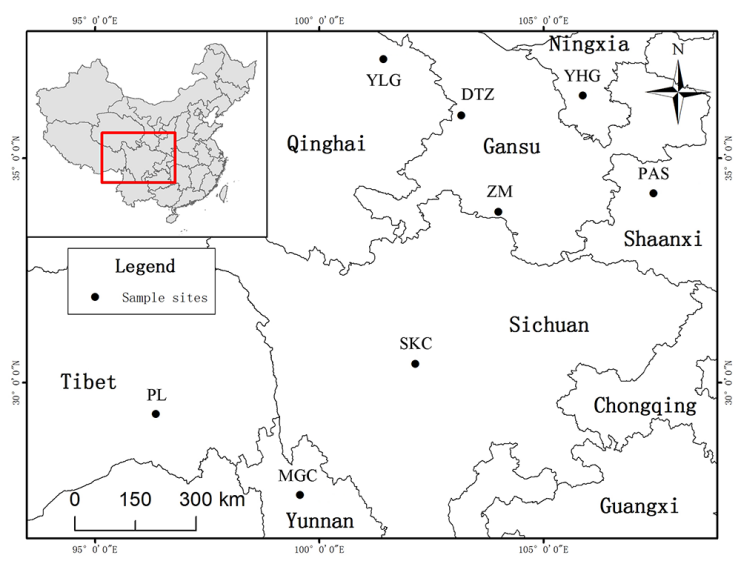

Figure 1. Locations of the eight Sinopodophyllum hexandrum populations sampled in this study.

Table 1. Information on the sampled Sinopodophyllum hexandrum populations.

\begin{tabular}{l|l|l|l|l|l|c|l|l}
\hline No. & Locations & Population & Code & Coordinates & N & Altitude $(\mathrm{m})$ & SO/SD $/{ }^{\circ}$ & Vegetation type \\
\hline 1 & Jingyuan, Ningxia & Yehegu & YHG & E106guan, Ningx & 20 & 2370 & SE40 & Forest-edge, shrub-grass \\
\hline 2 & Meixian, Shaanxi & Pingansi & PAS & E107ansi Shaan & 20 & 2815 & N75 & Shrub-grass \\
\hline 3 & Huzhu, Qinghai & Yuanlonggou & YLG & E102longgouhaix & 20 & 3069 & SW65 & Shrub-grass \\
\hline 4 & Yongdeng, Gansu & Datanzi & DTZ & E102nzig, Gansu & 20 & 2530 & NW75 & Forest-edge, shrub-grass \\
\hline 5 & Kangding, Sichuan & Shengkangcun & SKC & E102gkangcunc & 20 & 3207 & SW75 & Shrub-grass \\
\hline 6 & Shangri-la, Yunnan & Mugaocun & MGC & E99aocunla, Yu & 20 & 2250 & S 80 & Meadow, edge of forest \\
\hline 7 & Nyingchi, Tibet & Pula & PL & E94agchi, Tibe & 20 & 3256 & SE60 & Cliff, edge of forest \\
\hline 8 & Diebu, Gansu & Zemo & ZM & E103u, Gansuof & 20 & 2728 & SW30 & Forest-edge, shrub-grass \\
\hline
\end{tabular}

\section{Primer screening and ISSR-PCR amplification}

A total of 100 ISSR primers (synthesized by Shanghai Sheng Gong Biotechnology CO. LTD, China) were screened based on sequences from the Biotechnology Laboratory, University of British Columbia, Canada (UBC set No. 9) and the study of S. hexandrum (Xiao et al., 2006a,b; Alam et al., 2008; Alam et al., 2009; Naik et al., 2010; Liu et al., 2014). The reaction system was optimized by screening DNA, $\mathrm{Mg}^{2+}$, dNTP, primer (UBC900 was used for preliminary test), Taq DNA polymerase concentrations, annealing temperatures, and reaction conditions. The optimization showed that a $20-\mu \mathrm{L}$ reaction system was ideal under the following cycling profile: initial $5 \mathrm{~min}$ at $94^{\circ} \mathrm{C}$, followed by 45 cycles of $30 \mathrm{~s}$ at $94^{\circ} \mathrm{C}, 45 \mathrm{~s}$ annealing at $50^{\circ} \mathrm{C}$, and $90 \mathrm{~s}$ extension at $72^{\circ} \mathrm{C}$, ending with a final extension of 7 min at $72^{\circ} \mathrm{C}$. Each $20-\mu \mathrm{L}$ amplification reaction contained $1 \mathrm{X}$ PCR buffer $(10 \mathrm{mM}$ Tris-HCl at $\mathrm{pH} 8.3,50 \mathrm{mM} \mathrm{KCl}, 0.001 \%$ gelatin, and $1.5 \mathrm{mM} \mathrm{MgCl}_{2}$ ), $1.6 \mathrm{mM}$ dNTP Mix, $0.6 \mu \mathrm{M}$ primer (UBC900 was used for preliminary test), $15 \mathrm{ng}$ template DNA, and 1.0 U Taq DNA 
polymerase (TaKaRa Biotechnology, Dalian, China). Primer screening was performed in a PTC100TM Programmable Thermal Controller (MJ Research, Waltham, MA, USA) using the optimized PCR conditions. Three populations (PAS, YLG, and PL) were selected to screen 100 ISSR primers, using 10 samples per population. Primers that generated scorable bands and high levels of polymorphism were selected by genotyping all populations (rescreening the previous primers). The amplified products were electrophoresed on $1.0 \%$ agarose gels buffered with 1.0X TBE for $2.5 \mathrm{~h}$ at $100 \mathrm{~V}$. Bands were detected by ethidium bromide staining, and gels were imaged in the Gene Genius Bioimaging System. The size of each band was estimated from a 0.1-kbp DNA ladder (TaKaRa Biotechnology, Dalian, China). Amplification of each primer was performed in triplicate and a reaction without DNA was used as a negative control.

\section{Data analyses}

Amplification results were scored according to the position of the DNA bands generated by electrophoresis, being labeled " 1 " for the appearance of bands and " 0 " for the absence of bands in the data matrix. The resulting data matrix (binary matrix from 0 to 1 ) of the ISSR phenotype was analyzed using POPGENE software (version 1.31) (Yeh et al., 1997) to compute the following genetic diversity parameters: percentage of polymorphic bands (PPB), number of alleles per locus $\left(\mathrm{A}_{\mathrm{o}}\right)$, effective number of alleles per locus $\left(\mathrm{A}_{\mathrm{e}}\right)$, total gene diversity $\left(\mathrm{H}_{\mathrm{t}}\right)$, gene diversity within population $\left(\mathrm{H}_{\mathrm{s}}\right)$, coefficient of population differentiation $\left(\mathrm{G}_{\mathrm{st}}\right)$, the level of gene flow $\left(\mathrm{N}_{\mathrm{m}}\right)$, Nei's genetic diversity $\left(H_{\mathrm{E}}\right)$, Nei's genetic distance (D), Nei's genetic identity $(\mathrm{I})$, and Shannon's information index $\left(H_{\mathrm{O}}\right)$. POPGENE was also used to calculate fixation indexes $\left(F_{\mathrm{ST}}\right)$ between all pairwise combinations of populations. To examine genetic relationships at the species level, a dendrogram was constructed using the unweighted pair groups mean arithmetic (UPGMA) analysis in NTSYSpc2.10e software (Jensen, 1989). A bootstrap (resampling) test was performed 1000 times to obtain bootstrap values using PHYLIP version 3.69 (PHYLogeny Inference Package) program (Felsenstein, 1985). To visualize the genetic relationships among all ISSR phenotypes, their Euclidean distance matrix was subjected to a principle coordinate analysis (PCoA) using the program MVSP, version 1.3 (Kovach, 1999). Analysis of molecular variance (AMOVA) was performed to partition the total phenotypic variance of the entire data set into within- and among-population components using Arlequin 3.5.1.2 (Excoffier et al., 2005). The level of significance was tested by comparing the frequency distributions from the original dataset with the data generated by 1000 computer simulations. A Mantel test was performed to test isolation by distance (IBD) among populations by comparing genetic distance ( $F_{\mathrm{ST}}$ values) versus geographical distances using Tools for Population Genetic Analysis (TFPGA) (Miller, 1997), computing 5000 permutations.

\section{RESULTS}

\section{Genomic DNA amplification}

To investigate polymorphisms in $160 \mathrm{~S}$. hexandrum individuals from eight populations, 38 ISSR primers were chosen from the initial set of 100. PCR amplification (Table 2) showed that 11 primers produced a total of 141 clear and reproducible bands 
(250-2000 bp), of which 53 were polymorphic (a polymorphic rate of 37.59\%). Individual primers detected between 10 (UBC825) and 19 (UBC900) loci with an average of 12.82 clear amplified bands. Primer No. 11 (UBC900) amplified the highest number of bands (19) as well as the highest number of polymorphic bands (10), followed by primer No. 10 (UBC895). The percentage polymorphism ranged from 25\% (UBC834) to 52.63\% (UBC900), indicating that the selected primers were highly polymorphic across $S$. hexandrum populations. The $\mathrm{H}_{\mathrm{t}}$ was 0.1223 , whereas $\mathrm{H}_{\mathrm{s}}$ was found to be 0.0378 . The $\mathrm{G}_{\mathrm{st}}$ value of 0.6012 indicated that $39.88 \%$ of the genetic diversity resided within the populations. The $\mathrm{N}_{\mathrm{m}}$ among the sampled populations was calculated as 0.3317 using $\mathrm{G}_{\mathrm{st}}$ through the formula $\left(0.5\left[1-\mathrm{G}_{\mathrm{st}}\right] / \mathrm{G}_{\mathrm{st}}\right)$.

Table 2. Description of the 11 selected primers used for inter-simple sequence repeat (ISSR) amplification.

\begin{tabular}{l|l|l|c|c|c|c|c|c}
\hline No. & Primer & Sequence 5' $\rightarrow 3^{\prime}$ & $\mathrm{N}_{\mathrm{t}}$ & $\mathrm{N}_{\mathrm{p}}$ & $\mathrm{P}_{\mathrm{r}} \%$ & $\mathrm{H}_{\mathrm{t}}$ & $\mathrm{H}_{\mathrm{s}}$ & $\mathrm{G}_{\mathrm{st}}$ \\
\hline 1 & UBC825 & (AC)8T & 10 & 4 & 40.00 & 0.1504 & 0.0614 & 0.5321 \\
\hline 2 & UBC834 & (AG)8YT & 12 & 3 & 25.00 & 0.0824 & 0.0219 & 0.5937 \\
\hline 3 & UBC844 & (CT)8AGC & 13 & 4 & 30.77 & 0.0949 & 0.0296 & 0.5732 \\
\hline 4 & UBC845 & (CT)8AGG & 14 & 5 & 35.71 & 0.0981 & 0.0273 & 0.6044 \\
\hline 5 & UBC853 & (CT)8AGT & 11 & 3 & 27.27 & 0.091 & 0.0131 & 0.7047 \\
\hline 6 & UBC855 & (AC)8YT & 12 & 4 & 33.33 & 0.1136 & 0.0424 & 0.5401 \\
\hline 7 & UBC857 & (AC)8CTG & 11 & 5 & 45.45 & 0.1552 & 0.0542 & 0.5861 \\
\hline 8 & UBC867 & (GGC)6 & 10 & 3 & 30.00 & 0.104 & 0.0343 & 0.5681 \\
\hline 9 & UBC873 & (GACA)4 & 13 & 4 & 30.77 & 0.0954 & 0.022 & 0.6403 \\
\hline 10 & UBC895 & AGAGTTGGTAGCTCTTGATC & 16 & 8 & 50.00 & 0.1664 & 0.0544 & 0.6109 \\
\hline 11 & UBC900 & ACTTCCCCACAGGTTAACACA & 19 & 10 & 52.63 & 0.1939 & 0.0552 & 0.6593 \\
\hline Sum & & & 141 & 53 & & & & \\
\hline Mean & & & 4.82 & 37.59 & 0.1223 & 0.0378 & 0.6012 \\
\hline
\end{tabular}

$\mathrm{N}_{\mathrm{t}}=$ No. of total amplified bands; $\mathrm{N}_{\mathrm{p}}=$ No. of polymorphic bands; $\mathrm{P}_{\mathrm{r}}=$ polymorphism rate; $\mathrm{H}_{\mathrm{t}}=$ total gene diversity among population; $\mathrm{H}_{\mathrm{s}}=$ within population diversity; $\mathrm{G}_{\mathrm{st}}=$ mean coefficient of gene differentiation.

\section{Population genetic diversity}

Detailed statistical analyses were carried out based on the ISSR amplification results (Table 3 ). The highest level of genetic variability was observed in the PL population (PPB, 24.32\%) from Nysingchi, Tibet, whereas the PAS population from Mei County, Shaanxi showed the lowest variability (PPB, 7.66\%) among the eight populations. At the species level, the PPB was $37.59 \%$, whereas that of the single populations ranged from 7.66 (PAS) to $24.32 \%$ (PL), with an average of $17.12 \%$. A ranged from 1.0182 (PAS) to 1.1088 (SKC), with 1.1642 at the species level and 1.0658 at the population level. $H_{\mathrm{E}}$ was estimated to be 0.0963 at the species level and 0.0366 within populations. Within each population, the $H_{\mathrm{E}}$ ranged from 0.0141 to 0.0448 . High $H_{\mathrm{E}}(0.0573$, Table 3$)$ was observed in the MGC population only. $H_{\mathrm{O}}$ ranged from 0.0244 to 0.0961 , and the average $H_{\mathrm{O}}$ at the species level (0.2232) was higher than that at the population level (0.0607), showing that the genetic variation across populations was larger than that within any given population. To further analyze the population genetic diversity, Nei's genetic distance (D) and genetic identity (I) were quantified (Table 4) using POPGENE software. The largest D value was observed between PAS and PL, whereas the lowest D value was observed between YLG and DTZ, indicating that the highest genetic identity was found between YLG and DTZ and the lowest was found between PAS and PL. 
Table 3. Genetic diversity parameters for eight populations of Sinopodophyllum hexandrum.

\begin{tabular}{l|c|c|c|c|c}
\hline Population & PPB (\%) & $\mathrm{A}_{\circ}$ & $\mathrm{A}_{\mathrm{e}}$ & $H_{\mathrm{E}}$ & $H_{\mathrm{O}}$ \\
\hline YHG & 11.12 & 1.1088 & 1.0826 & 0.0315 & 0.0533 \\
\hline PAS & 7.66 & 1.0761 & 1.0182 & 0.0141 & 0.0244 \\
\hline YLG & 15.43 & 1.1253 & 1.0611 & 0.0412 & 0.0431 \\
\hline DTZ & 17.33 & 1.1576 & 1.0445 & 0.0226 & 0.0656 \\
\hline SKC & 18.04 & 1.1842 & 1.1088 & 0.0387 & 0.0603 \\
\hline MGC & 20.63 & 1.2165 & 1.0802 & 0.0573 & 0.0961 \\
\hline PL & 24.32 & 1.2577 & 1.0735 & 0.0422 & 0.0722 \\
\hline ZM & 22.44 & 1.2435 & 1.0573 & 0.0448 & 0.0704 \\
\hline Average & 17.12 & 1.1712 & 1.0658 & 0.0366 & 0.0607 \\
\hline Total & 37.59 & 1.4768 & 1.1642 & 0.0963 & 0.2232 \\
\hline
\end{tabular}

$\mathrm{A}_{\mathrm{o}}=$ observed number of alleles per locus; $\mathrm{A}_{\mathrm{e}}=$ effective number of alleles per locus; $H_{\mathrm{E}}=$ expected heterozygosity; $H_{\mathrm{O}}=$ Shannon's information index; $\mathrm{PPB}=$ percentage of polymorphic loci.

Table 4. Nei's genetic identity and Nei's genetic distance between eight populations of Sinopodophyllum hexandrum.

\begin{tabular}{l|c|c|c|c|c|c|c|c}
\hline Population & YHG & PAS & YLG & DTZ & SKC & MGC & PL & ZM \\
\hline YHG & - & 0.9638 & 0.9288 & 0.9244 & 0.9167 & 0.9346 & 0.9468 & 0.9257 \\
\hline PAS & 0.0362 & - & 0.9421 & 0.9356 & 0.9367 & 0.9427 & 0.8880 & 0.9164 \\
\hline YLG & 0.0712 & 0.0579 & - & 0.9736 & 0.9457 & 0.9488 & 0.9545 & 0.9632 \\
\hline DTZ & 0.0756 & 0.0644 & 0.0264 & - & 0.9533 & 0.9487 & 0.9432 & 0.9236 \\
\hline SKC & 0.0833 & 0.0633 & 0.0543 & 0.0467 & - & 0.9186 & 0.9478 & 0.9433 \\
\hline MGC & 0.0654 & 0.0573 & 0.0512 & 0.0513 & 0.0814 & - & 0.9366 & 0.9246 \\
\hline PL & 0.0532 & 0.1120 & 0.0455 & 0.0568 & 0.0522 & 0.0634 & - & 0.9178 \\
\hline ZM & 0.0743 & 0.0836 & 0.0368 & 0.0764 & 0.0567 & 0.0754 & 0.0822 & - \\
\hline
\end{tabular}

Nei's genetic identity (I) above diagonal; Nei's genetic distance (D) below diagonal.

\section{Genetic structure and differentiation of the populations}

\section{UPGMA cluster analysis}

An UPGMA cluster-dendrogram (Figure 2) was constructed to investigate the genetic relationship between the eight populations. Three clusters were defined among eight populations at a genetic distance of $0.08-0.10$. Cluster A contained only population PL, which is located in Tibet. Tibet has unique climatic and geographical conditions, and the field observations showed that PL differed from the other populations and had moderate plant height, exquisite leaves, and good root texture. Cluster B contained five populations, which could be subdivided into three clusters: (i) $\mathrm{B}_{1}$, with population ZM; (ii) $\mathrm{B}_{2}$, with populations DTZ and YLG; and (iii) $\mathrm{B}_{3}$, with populations YHG and PAS. The plant height of these populations was very high. They had similar habitat conditions and plant morphological characteristics because the plants were collected from the edges of the forests and river valleys. Cluster $\mathrm{C}$ contained two populations, SKC and MGC. These materials were collected from high altitude regions. Plant height was the tallest, but the leaves had purple plaques likely due to the strong ultraviolet radiation. Their populations were concentrated in a schistose pattern, possessing rough leaves and medium root texture. The $S$. hexandrum population was not clustered according to the geographic distance on the UPGMA tree, which may indicate high genetic differentiation between populations. 


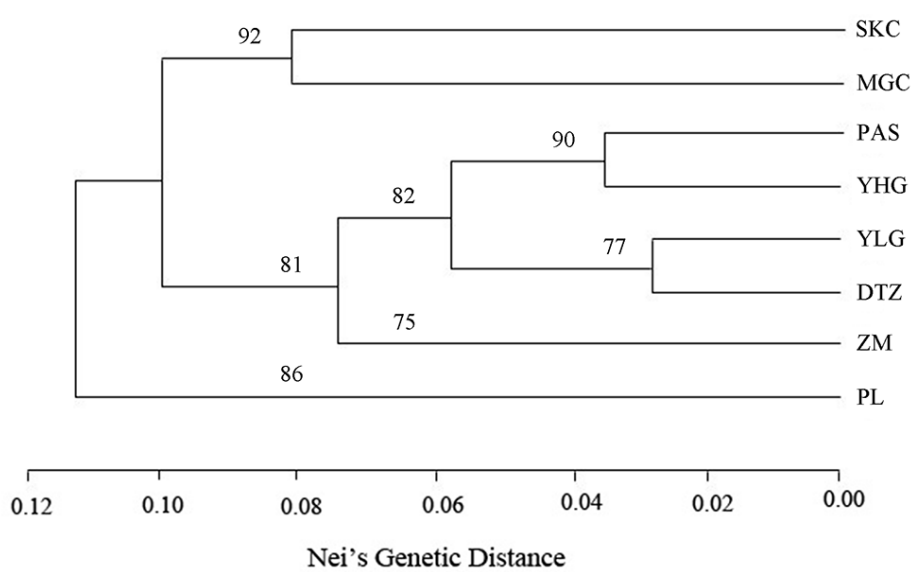

Figure 2. Unweighted pair groups mean arithmetic (UPGMA) clustering of Sinopodophyllum hexandrum populations.

\section{Principle coordinate analysis (PCoA)}

PCoA was used for ordination and exploration of the similarity between populations. The plot of the first (PCo1) and second ( $\mathrm{PCo} 2)$ principal coordinates from a PCoA is depicted in Figure 3. All samples were clearly separated into three major groups on the PCo1 and PCo2. PCo1 accounted for $25.69 \%$ of the total variance, and PCo2 accounted for $11.76 \%$. All sampled individuals were strongly assigned to their original populations. The clustering of the populations was consistent with the UPGMA dendrogram, signifying the hierarchical relationships of the populations and genetic groups.

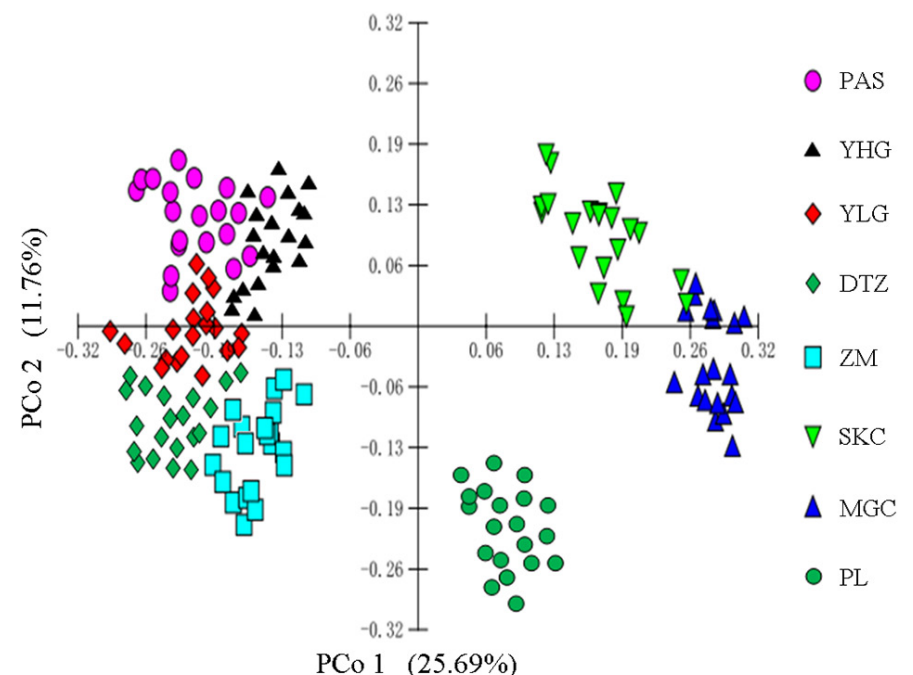

Figure 3. Distribution of individuals from the eight Sinopodophyllum hexandrum populations according to the first principal coordinate (PCo1) and second principal coordinate (PCo2). PCo1 and PCo2 account for 25.69 and $11.76 \%$ of the total variation, respectively. 


\section{Mantel test for IBD}

A Mantel test for IBD was performed to investigate the correlation between genetic distance $\left(F_{S T}\right)$ and the corresponding geographic distance of eight $S$. hexandrum populations. The correlation coefficient $r$ of genetic distance and geographic distance was $0.212(\mathrm{P}=$ 0.99), and the correlation analysis diagram (Figure 4) comprised many disordered and scattered points, indicating that the IBD of $S$. hexandrum populations is not significant at the level of 0.05 . The Mantel test showed revealed no correlation between genetic distance and geographical provenance of $S$. hexandrum populations. This result is consistent with the conclusions made by Xiao et al. (2006a,b).

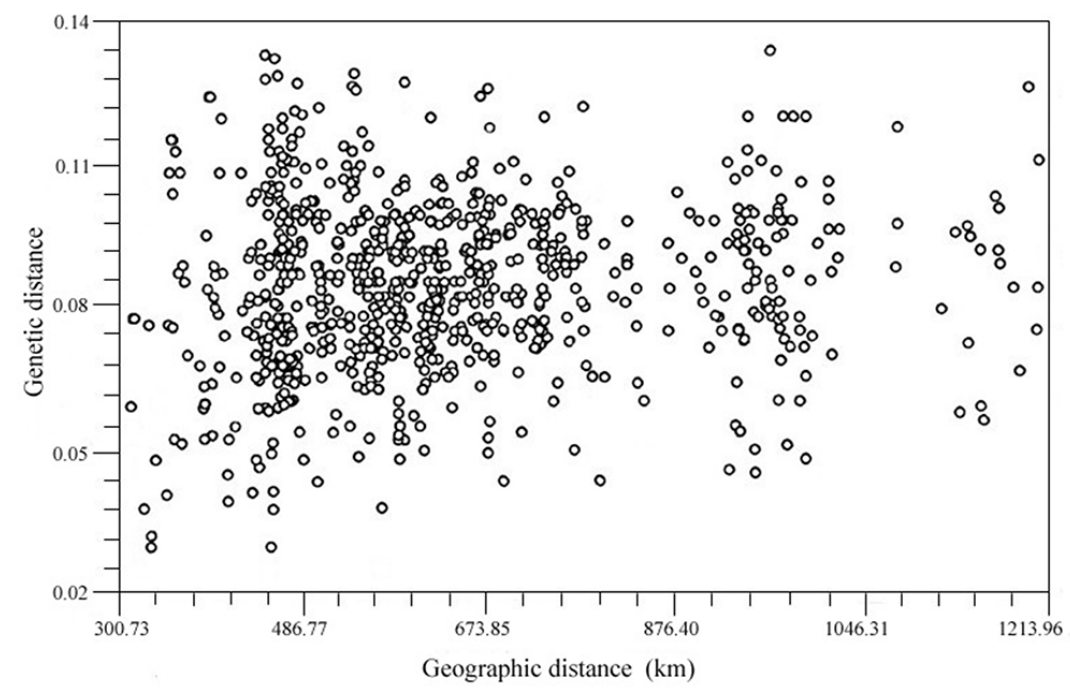

Figure 4. Mantel regression of the pairwise relationship between genetic $\left(F_{S T}\right)$ and geographical distances for Sinopodophyllum hexandrum populations.

\section{Analysis of molecular variance (AMOVA)}

Prior to AMOVA, the coefficient of population differentiation $\left(\mathrm{G}_{\mathrm{st}}=0.6012\right.$, Table 2) for $S$. hexandrum was calculated with POPGENE software. Overall, $60.12 \%$ of the total genetic variation existed among the populations, and only $39.88 \%$ of the genetic variation resided within the populations, illustrating that higher genetic differentiation occurred between the populations. AMOVA was performed to further evaluate genetic structure (Table 5). First, the three major groups defined according to the results of UPGMA and PCoA were tested. As expected, variance among the populations was highly significant $(\mathrm{P}<0.0002)$ and explained $63.27 \%$ of the total genetic variance, supporting the results of the hierarchical clustering and PCoA. The variance within populations only explained $36.73 \%$ of the total, indicating that the level of genetic differentiation between the populations was higher than that within each population. This result was consistent with the calculated $\mathrm{G}_{\mathrm{st}}$. 
Table 5. Hierarchical analysis of molecular variance (AMOVA) for eight populations of Sinopodophyllum hexandrum.

\begin{tabular}{l|c|c|c|c|c|c}
\hline Source of variation & d.f. & Sum of squares & Mean squares & Variance component & Total variance (\%) & P value \\
\hline Among population & 7 & 602.323 & 99.946 & 4.9128 & 63.27 & $<0.0002$ \\
\hline Within population & 128 & 99.766 & 2.964 & 2.9621 & 36.73 & $<0.0002$ \\
\hline
\end{tabular}

\section{DISCUSSION}

\section{Genetic diversity of $S$. hexandrum}

ISSR markers were used to amplify genomic DNA with universal primers, which were designed to detect variations in microsatellite sequences. Organisms accumulate abundant microsatellite variations during long-term evolution because these sequences do not encode functional genes. Furthermore, there are only small changes in the number of repeat units throughout the entire organism, which shows that ISSR markers harbor higher levels of polymorphism (Cruzan, 1998). Comparing the average genetic diversity with that of S. hexandrum from other regions (Xiao et al., 2006b; Alam et al., 2008; Naik et al., 2010; Liu et al., 2014) and with other Berberidaceae species (Qiu et al., 2006; Zong et al., 2008) based on the ISSR approach showed that $S$. hexandrum populations have low genetic diversity (average $H_{\mathrm{E}}=0.0366$ ). The $S$. hexandrum populations from the Northwestern Himalayan region (Xiao et al., 2006b; Alam et al., 2008; Naik et al., 2010) (average $H_{\mathrm{E}}=0.2944,0.092$, respectively) and Qinling Mountains (average $H_{\mathrm{E}}=0.0621$ ) showed high genetic diversity. $S$. hexandrum is native to the Himalayan region, where it grows in valleys with secondary vegetation, under shrubs, or around trees (Ma and $\mathrm{Hu}, 1996)$. The Qinling Mountains are a 1500-km mountain chain that runs east-west and divides the watershed between the Yangtze River and the Yellow River in China, which constitute a transitional zone between the northern subtropical zone and the warmtemperate zone. The habitat in this region differs significantly from that of our study sites.

$S$. hexandrum reproduces through vegetative reproduction and seeds. Insects and birds are limited to the high altitude regions, implying that $S$. hexandrum pollination is more difficult in the Himalayan region and Qinling Mountains. Another Berberidaceae species, Dysosma versipellis, is an endangered species endemic to China, which has been listed as a key protected wild plant in China due to habitat fragmentation and destruction. Qiu et al. (2006) found this species to have a high level of genetic diversity in China $\left(H_{\mathrm{E}}=0.378\right)$. Dysosma pleiantha, a threatened medicinal plant species distributed in southeastern China, reproduces both sexually and asexually and was found to have a high $H_{\mathrm{E}}(0.364)$ (Zong et al., 2008). The relatively high level of genetic variation observed within these two species suggested that the balance between vegetative reproduction and sexual reproduction favored sexual reproduction in the $D$. versipellis and $D$. pleiantha populations, as compared with the $S$. hexandrum populations. The $\mathrm{H}_{\mathrm{e}}$ found in this study, 0.0963 at the species level, was lower than that found in some strictly self-pollinating soybean species $\left(H_{\mathrm{E}}=0.1714\right)$ (Jin et al., 2003) and the self-pollinating Oryza granulata $\left(H_{\mathrm{E}}=0.210\right)$ (Wu et al., 2004), which also indicated that potential self-pollination in these populations led to a reduction in their genetic diversity.

Genetic diversity is affected by multiple factors, such as geographical distribution, mating system, life form, and pollen and seed dispersal (Ohsawa et al., 2008). Wild populations of $S$. hexandrum are very small and decline each year in China because of habitat fragmentation and human exploitation. The rapid decline in the number of individuals in wild populations 
may not cause an immediate decrease in the genetic diversity of the species. However, when all populations are under pressure and the species vegetative reproduction capacity predominates, it may lead to an overall decrease in genetic diversity, resulting in a decrease in the long-term adaptability to environmental changes. In the present study, the PL population from Nyingchi, Tibet had the highest genetic diversity, followed by populations from Diebu, Gansu (ZM), with the lowest genetic diversity found for the PAS population from Mei County, Shaanxi. Notably, the field survey showed that the habitats of PL and ZM populations were well-protected. Conversely, the PAS population was located within tourist areas of the Taibai Mountain, and its habitat has been almost entirely destroyed by human activity. Therefore, all of the remaining plants grew under miscellaneous forest trees in strong sunlight, and the populations grew weakly and recessed. This may explain why the PAS population has relatively low genetic diversity. Historical events are also responsible for variation in genetic diversity (Karron, 1991). S. hexandrum is native to the Himalayan region, which includes China, India, Nepal, and Myanmar. Further research should include populations from other regions of China, and from other countries.

\section{High genetic differentiation and distinct genetic structure}

High levels of genetic differentiation and clear population structure were detected for $S$. hexandrum in this study. All sampled individuals were strongly assigned to their original populations, and all analyses (UPGMA and PCoA) strongly supported these eight $S$. hexandrum populations distributed in China being clustered into three major groups. These methods consistently showed that the genetic differentiation of $S$. hexandrum was higher among than within populations, which is consistent with the findings for genetic variation in certain selfing species (Nybom and Bartish, 2000). This means that $S$. hexandrum should be a selfing species or a selfing predominant species, which is consistent with previous results for $S$. hexandrum (Ma et al., 1997). Aside from the breeding system, the high level of genetic differentiation across populations may be caused by genetic drift (Hamrick and Godt, 1989). Wright (1965) noted that genetic drift would lead a small population to emerge with distinct genetic differentiation when the $\mathrm{N}_{\mathrm{m}}$ value was lower than 1.0. The $\mathrm{N}_{\mathrm{m}}$ of $S$. hexandrum (0.3317) obtained using POPGENE was lower than 1.0 in the present study, which suggests that some genetic drift may have emerged among the populations of this species. The distribution of $S$. hexandrum populations revealed clear fragmentation based on the field investigation, which is consistent with the possibility of genetic drift.

Factors such as fragmented geographical distribution and a lack of pollinators or seed dispersers can be a barrier to gene flow between populations (Zhou et al., 2010). Limited gene flow $\left(\mathrm{N}_{\mathrm{m}}=0.3317\right)$ among $S$. hexandrum populations may be related to the dominant reproductive mechanism and inbreeding in this species, as well as the limited seed propagation distance. Some studies have found that seed dispersal is a primary factor influencing variation in gene flow and population structure (Kalisz et al., 1999). Mature berries from S. hexandrum usually fall to the ground because of rain or wind, resulting in the presence of some seed residue in the soil, whereas others are dispersed by cattle, birds, or humans ( $\mathrm{Ma}$ and $\mathrm{Hu}, 1996$ ). Therefore, the short seed dispersal distance of $S$. hexandrum probably resulted in limited gene flow among populations. Mountain ranges and rivers are possible barriers to both dispersal of pollen or rhizomes of $S$. hexandrum, which reproductively isolates the populations. Furthermore, the Mantel test revealed that the genetic distance between populations did not 
correlate with the geographical distance. Restricted gene flow associated with geographical distance is consistent with the results of previous studies on this species (Alam et al., 2008; Alam et al., 2009; Naik et al., 2010).

\section{Implications for conservation}

S. hexandrum is considered a rare and threatened species in China ( $\mathrm{Fu}, 1991)$. Assessing genetic diversity is important for designing conservation strategies for threatened and endangered species (Hamrick and Godt, 1989). The results of the present study show that $S$. hexandrum populations possess low levels of genetic diversity, and that genetic differentiation among populations is higher than that within populations. The loss of genetic diversity usually has deleterious effects on species fitness and may threaten the survival of populations, and could therefore account for the endangered status of S. hexandrum in China (Reed and Frankham, 2003). Estimates of genetic diversity and population genetic structure could provide a basis for the conservation and utilization of the endangered $S$. hexandrum. Furthermore, it will help us to determine what should be conserved and where and how this can be achieved.

The field survey of natural $S$. hexandrum populations showed that this species has been over-collected because of its medical value, and that the habitats of some populations have subsequently been destroyed. In particular, in the case of the YHG (Jingyuan, Ningxia) and PAS (Mei County, Shaanxi) populations, the habitats are not suitable for the growth of this species. Damage to natural habitats would lead to a decrease in population size and a possible increase in inbreeding, resulting in a loss of genetic diversity. Therefore, it is very important to maintain a stable habitat that is suitable for $S$. hexandrum populations. In situ conservation effectively and sustainably addresses this problem. Establishing $S$. hexandrum reserves should be the primary method of conservation. The wild populations (PL, ZM, and MGC) with relatively high levels of genetic diversity should be conserved as a priority in this setting because limited gene flow exists among populations of $S$. hexandrum. Establishing reserves may be profitable for the PL, ZM, and MGC populations because these are situated in state forest conservation areas in China, which are not subjected to cutting and hunting Mixing highly divergent populations may be risky, because it could result in the loss of adaptive diversity (Tang et al., 2014). Therefore, it is necessary to improve gene flow among populations within each group through some artificial means, such as transplanting individuals (by seed or rhizomes from one population to another). In addition, to avoid over-collection, greater awareness must be emphasized, and local inhabitants should be motivated to cultivate medicinal plants as vicarious species, and cultivation packages of the medicinal plant must be developed for the local inhabitants. State forest departments, nongovernmental organizations, village committees, and voluntary bodies should be encouraged and suitably funded to undertake conservation through clonal propagation and other measures.

The second management strategy is a program of ex situ conservation to supplement in situ conservation. Populations may be partially preserved through seed banks or in vitro germplasm collections. S. hexandrum has good sexual reproduction. Each plant can produce approximately 60 seeds, with a maximum of approximately 180 (Ma et al., 1997). Seeds can be easily collected from $S$. hexandrum compared with other endangered species. Thus, a strategy involving extensive collection to ensure full sampling of genetic diversity, subsequent cultivation in a garden at least $1000 \mathrm{~m}$ above sea level, and reintroduction into the wild, seems feasible. For $S$. hexandrum populations in China, there are some preserved forest farms, which 
could be used for relocation. However, ex situ conservation has many drawbacks because it is impossible to fully recreate the natural habitat. The new environment may have important ecological differences compared with the original habitat, and artificially propagated plants adopting local seed sources are more likely to exhibit increased fitness over non-local genotypes. Therefore, seedlings should be selected from different populations and then be transplanted into their original localities. The ex situ conservation approach is technically challenging, often expensive, and recommended only to supplement in situ conservation, or as a last resort. In addition, in vitro techniques have proven to be an alternative and effective means of propagation that can facilitate the recovery of rare and endangered species (Nadeem et al., 2000). At present, an effective protocol of in vitro propagation, involving the formation of multiple shoots from zygotic embryos and subsequent rooting, could be used for $S$. hexandrum. Although in vitro techniques are known to induce variability, plants raised through tissue culture can be screened for useful somaclonal variants and then exploited to obtain plants or cultures with high podophyllotoxin content, possibly reducing the pressure on natural populations of $S$. hexandrum.

By using knowledge on the distribution of genetic diversity and population clustering, it is possible to design the best sampling and conservation strategies to maintain diversity in this important medicinal and endangered species.

\section{Conflicts of interest}

The authors declare no conflict of interest.

\section{ACKNOWLEDGMENTS}

Research supported by the program from the Forestry Research Foundation for the Public Service Industry of China (\#200904004). We are also grateful to Genlu Bai, Guowei $\mathrm{Xia}$ and all the colleagues in the same laboratory for the assistance in the work.

\section{REFERENCES}

Alam A, Naik PK, Gulati P, Gulati AK, et al. (2008). Characterization of genetic structure of Podophyllum hexandrum populations, an endangered medicinal herb of northwestern Himalaya, using ISSR-PCR markers and its relatedness with podophyllotoxin content. Afr. J. Biotechnol. 7: 1028-1040.

Alam MA, Gulati P, Gulati AK, Mishra GP, et al. (2009). Assessment of genetic diversity among Podophyllum hexandrum genotypes of the North-Western Himalayan region for podophyllotoxin production. Indian J. Biotechnol. 8: 391-399.

Bornet B and Branchard M (2001). Nonanchored inter simple sequence repeat (ISSR) markers: Reproducible and specific tools for genome fingerprinting. Plant Mol. Biol. Rep. 19: 209-215. http://dx.doi.org/10.1007/BF02772892

Chattopadhyay S, Bisaria VS, Panda AK and Srivastava AK (2004). Cytotoxicity of in vitro produced podophyllotoxin from Podophyllum hexandrum on human cancer cell line. Nat. Prod. Res. 18: 51-57. http://dx.doi. org $/ 10.1080 / 1057563031000122095$

Cragg GM, Newman DJ and Snader KM (1997). Natural products in drug discovery and development. J. Nat. Prod. 60: 52-60.http://dx.doi.org/10.1021/np9604893

Cruzan MB (1998). Genetic markers in plant evolutionary ecology. Ecology 79: 400-412. http://dx.doi.org/10.1890/00129658(1998)079[0400:GMIPEE]2.0.CO;2

Editorial Committee of Flora of China (2011). Flora of China (Vol. 19). Science Press \& Missouri Botanical Garden Press, Beijing \& St. Louis.

Excoffier L, Laval G and Schneider S (2005). Arlequin (version 3.0): an integrated software package for population genetics data analysis. Evol. Bioinform. Online 1: 47-50. 
Felsenstein J (1985). Confidence limits on phylogenies: An approach using the bootstrap. Evolution 39: 783-791. http:// dx.doi.org/10.2307/2408678

Fu LG (1991). Plant red book of China: Rare threatened plant. Science Press, Beijing.

Giri A and Lakshmi Narasu M (2000). Production of podophyllotoxin from Podophyllum hexandrum: a potential natural product for clinically useful anticancer drugs. Cytotechnology 34: 17-26.http://dx.doi.org/10.1023/A:1008138230896

Hamrick JL and Godt MJW (1989). Allozyme diversity in plant species. in: plant population genetics, breeding and genetic resources (Brown AHD, Clegg MT, Khaler AL and Weir BS, eds.). Sinauer Associates, Inc. Publisher, Sunderland, 43-63.

Jensen RJ (1989). Ntsys-Pc-numerical taxonomy and multivariate analysis system-version 1.40. Q. Rev. Biol. 64: 250-252.

Jin Y, Zhang WJ, Fu DX and Lu BR (2003). Sampling strategy within a wild soybean population based on its genetic variation detected by ISSR markers. Acta Bot. Sin. 45: 995-1002.

Kalisz S, Hanzawa FM, Tonsor SJ, Thiede DA, et al. (1999). Ant-mediated seed dispersal alters pattern of relatedness in a population of Trillium grandiflorum. Ecology 80: 2620-2634. http://dx.doi.org/10.2307/177245

Karron JD (1991). Patterns of genetic variation and breeding systems in rare plant species (Falk DA and Holsinger KE, eds.). Genetics and Conservation of Rare Plants.Oxford University Press, New York, 87-98.

Kovach W (1999). MVSP-a multivariate statistical package for Windows, ver. 3.1. Kovach Computing Services, Pentraeth, Wales, UK.

Lata H, Moraes RM, Bertoni B and Pereira AMS (2010). In vitro germplasm conservation of Podophyllum peltatum L. under slow growth conditions. In Vitro Cell. Dev. Biol. Plant 46: 22-27. http://dx.doi.org/10.1007/s11627-009-9243-5

Li Y, Zhai SN, Qiu YX, Guo YP, et al. (2011). Glacial survival east and west of the 'Mekong-Salween Divide'in the Himalaya-Hengduan Mountains region as revealed by AFLPs and cpDNA sequence variation in Sinopodophyllum hexandrum (Berberidaceae), Mol. Phylogenet. Evol. 59: 412-424.

Liu W, Yin D, Liu J and Li N (2014). Genetic diversity and structure of Sinopodophyllum hexandrum (Royle) Ying in the Qinling Mountains, China. PLoS One 9: e110500.http://dx.doi.org/10.1371/journal.pone.0110500

Ma SB and Hu ZH (1996). Preliminary research on distribution pattern and the ecological adaption of Sinopodophullum hexandrum. J. Wuhan Bot. Res. 14: 47-54.

Ma SB, Xu ZR and Hu ZH (1997). A contribution to the reproductive biology of Sinopodophyllum hexandrum (Royle) Ying (Berberidaceae). Acta Bot. Boreal. -. Occid. Sin. 17: 49-55.

Miller MP (1997). Tools for population genetic analysis (TFPGA), Vesion 1.3: A Windows program for the analysis of allozyme and molecular population genetic data. Department of Biological Sciences, Northern Arizona University, Ariozna.

Nadeem M, Palni L, Purohit A, Pandey H, et al. (2000). Propagation and conservation of Podophyllum hexandrum Royle: An important medicinal herb. Biol. Conserv. 92: 121-129. http://dx.doi.org/10.1016/S0006-3207(99)00059-2

Naik PK, Alam MA, Singh H, Goyal V, et al. (2010). Assessment of genetic diversity through RAPD, ISSR and AFLP markers in Podophyllum hexandrum: a medicinal herb from the Northwestern Himalayan region. Physiol. Mol. Biol. Plants 16: 135-148. http://dx.doi.org/10.1007/s12298-010-0015-9

Nybom H and Bartish IV (2000). Effects of life history traits and sampling strategies on genetic diversity estimates obtained with RAPD markers in plants. Perspect. Plant. Ecol. 3: 93-114. http://dx.doi.org/10.1078/1433-8319-00006

Ohsawa T, Saito Y, Sawada H and Ide Y (2008). Impact of altitude and topography on the genetic diversity of Quercus serrata populations in the Chichibu Mountains, Central Japan. Flora 203: 187-196. http://dx.doi.org/10.1016/j. flora.2007.02.007

Qiu YX, Li JH, Liu HL, Chen YY, et al. (2006). Population structure and genetic diversity of Dysosma versipellis (Berberidaceae), a rare endemic from China. Biochem. Syst. Ecol. 34: 745-752. http://dx.doi.org/10.1016/j. bse.2006.06.001

Reed DH and Frankham R (2003). Correlation between fitness and genetic diversity. Conserv. Biol. 17: 230-237. http:// dx.doi.org/10.1046/j.1523-1739.2003.01236.x

Svensson B and Pettersson H (2003). Reumacon (CPH82) showed similar x-ray progression and clinical effects as methotrexate in a two year comparative study on patients with early rheumatoid arthritis. Scand. J. Rheumatol. 32: 83-88. http://dx.doi.org/10.1080/03009740310000085

Tang N, Mo G, Van Tuyl JM, Arens P, et al. (2014). Genetic diversity and structure of Lilium pumilum DC. in southeast of Qinghai-Tibet plateau. Plant Syst. Evol. 300: 1453-1464.

Wright S (1965). The interpretation of population structure by F-statistics with special regard to systems of mating. Evolution 19: 395-420. http://dx.doi.org/10.2307/2406450

Wu CJ, Cheng ZQ, Huang XQ, Yin SH, et al. (2004). Genetic diversity among and within populations of Oryza granulata from Yunnan of China revealed by RAPD and ISSR markers: Implications for conservation of the endangered species. Plant Sci. 167: 35-42. http://dx.doi.org/10.1016/j.plantsci.2004.02.022

Xiao M, Li Q, Guo L, Luo T, et al. (2006a). AFLP analysis of genetic diversity of the endangered species Sinopodophyllum hexandrum in the Tibetan region of Sichuan Province, China. Biochem. Genet. 44: 47-60. http://dx.doi.org/10.1007/ $\underline{\text { s10528-006-9006-5 }}$ 
Xiao M, Li Q, Wang L, Guo L, et al. (2006b). ISSR analysis of the genetic diversity of the endangered species Sinopodophyllum hexandrum (Royle) Ying from western Sichuan Province. China. J. Integr. Plant Biol. 48: 11401146. http://dx.doi.org/10.1111/j.1744-7909.2006.00304.x

eh FC, Yang RC, Boyle TB, Ye Z, et al. (1997). POPGENE: The user-friendly shareware for population genetic analysis. Molecular Biology and Biotechnology Centre, University of Alberta, Edmonton.

Zhao CQ, Zhu YY, Chen SY and Ogihara Y (2011). Lignan glucoside from Sinopodophyllum emodi and its cytotoxic activity. Chin. Chem. Lett. 22: 181-184. http://dx.doi.org/10.1016/j.cclet.2010.10.013

Zhou TH, Qian ZQ, Li S, Guo ZG, et al. (2010). Genetic diversity of the endangered chinese endemic herb Saruma henryi oliv. (Aristolochiaceae) and its implications for conservation. Popul. Ecol. 52: 223-231. http://dx.doi.org/10.1007/ $\underline{\text { s10144-009-0139-3 }}$

Zong M, Liu HL, Qiu YX, Yang SZ, et al. (2008). Genetic diversity and geographic differentiation in the threatened species Dysosma pleiantha in China as revealed by ISSR analysis. Biochem. Genet. 46: 180-196. http://dx.doi.org/10.1007/ $\underline{\text { s10528-007-9141-7 }}$ 